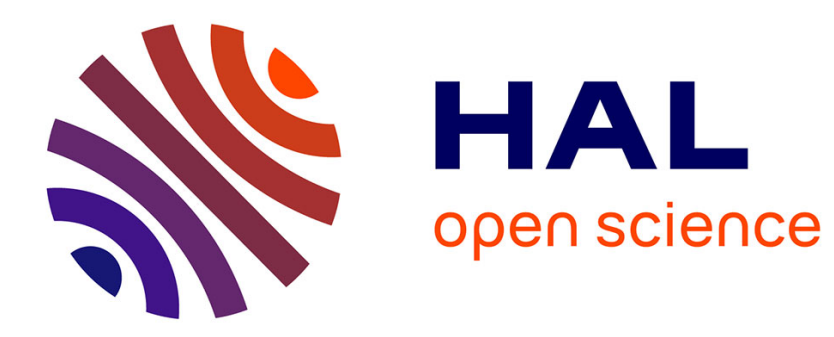

\title{
Diffraction et ordre local dans les amorphes. Cas des neutrons thermiques
}

\author{
G. Tourand
}

\section{To cite this version:}

G. Tourand. Diffraction et ordre local dans les amorphes. Cas des neutrons thermiques. Revue de Physique Appliquée, 1977, 12 (5), pp.631-636. 10.1051/rphysap:01977001205063100 . jpa-00244218

\section{HAL Id: jpa-00244218 https://hal.science/jpa-00244218}

Submitted on 1 Jan 1977

HAL is a multi-disciplinary open access archive for the deposit and dissemination of scientific research documents, whether they are published or not. The documents may come from teaching and research institutions in France or abroad, or from public or private research centers.
L'archive ouverte pluridisciplinaire HAL, est destinée au dépôt et à la diffusion de documents scientifiques de niveau recherche, publiés ou non, émanant des établissements d'enseignement et de recherche français ou étrangers, des laboratoires publics ou privés. 


\title{
DIFFRACTION ET ORDRE LOCAL DANS LES AMORPHES CAS DES NEUTRONS THERMIQUES
}

\author{
G. TOURAND \\ DPh-G/PSRM - C.E. N. Saclay, B.P. no 2, 91190 Gif-sur-Yvette, France
}

\begin{abstract}
Résumé. - Les systèmes désordonnés amorphes ont commencé à susciter l'intérêt des Physiciens et des Chimistes bien avant la découverte de leurs propriétés de commutation d'un état semiconducteur à un état métallique. La caractéristique essentielle de ces matériaux est bien évidemment la non-périodicité à longue distance pour des systèmes à l'état solide. D'un point de vue phénoménologique, un amorphe était défini comme un corps ne présentant pas de spectre de diffraction fin.

Nous tenterons de définir de façon plus rigoureuse cet état amorphe puis, dans le cadre des propriétés structurales, dont la connaissance est essentielle pour une meilleure compréhension de ces systèmes, nous rappellerons comment est définie la fonction de distribution de paire $g(r)$. Nous montrerons quelles sont les informations que cette fonction permet d'obtenir pour la connaissance de l'ordre local, et quelles sont les limites de son utilisation en fonction des approximations utilisées.

Nous examinerons ensuite brièvement les différentes méthodes d'investigation dont nous disposons pour obtenir cette fonction de corrélation de paire. Nous insisterons alors plus particulièrement sur le cas des neutrons thermiques en montrant la démarche suivie pour obtenir à partir de l'information expérimentale (intensité diffusée) la fonction de corrélation de paire $g(r)$ que nous utilisons comme paramètre d'étude de l'ordre à courte distance.
\end{abstract}

\begin{abstract}
The disordered amorphous systems have been of great interest for physicists and chemists for a long time before light was shed on the nature of the semiconductor-to-metal transition that occurs in these materials.

The essential feature of these systems is the lack of periodicity in the solid state. Phenomenologically an amorphous material was defined as a system without any well-defined diffraction pattern.

We shall attempt to give a more rigorous definition of the amorphous state. The pair distribution function $g(r)$ will then be defined with respect to the structural properties, a knowledge of which is essential for a better understanding of amorphous systems. The information yielded by this function on the nature of the local order and its limitations due to the various approximations used will be discussed.

The different experimental means available for obtaining the pair correlation function will be critically examined. Emphasis will be placed on thermal neutron measurements. Finally, we shall explain how the function $g(r)$ used as a local order parameter is deduced from the experimental data (diffuse intensity).
\end{abstract}

Les progrès réalisés dans l'étude des solides cristallins sont dus autant à la quantité et aux raffinements des recherches expérimentales qu'à l'élaboration de théories toujours plus évoluées. Mais tous les états de la matière n'ont pas bénéficié des mêmes avantages et le vaste domaine des systèmes désordonnés ou solides non cristallins en est un exemple.

Avant de tenter de définir ces substances peut-être n'est-il pas inutile de rappeler comment elles se situent parmi les états de la matière. En effet dès l'antiquité, il a été supposé que la matière était formée de quatre éléments qui étaient le ciel, la terre, l'eau et le feu. Les trois premiers éléments symbolisaient les états gazeux, solides et liquides et le rôle du feu consistait à faciliter les passages d'une forme de matière à une autre. Cette idée fondamentale des quatre éléments se perpétua fort longtemps puisqu'elle était encore à l'honneur au début $\mathrm{du} \mathrm{XVII}^{\mathrm{e}}$ siècle et les trois états de la matière dont on parle couramment de nos jours ne sont que l'héritage des quatre éléments d'autrefois.

Aux frontières de ces trois états de la matière des formes intermédiaires ont été mises en évidence, ce sont par exemple :

- les cristaux liquides qui possèdent la fluidité des liquides simples et les propriétés anisotropes des cristaux ;

- les corps amorphes, substances désordonnées à l'image d'un liquide, mais un liquide qui aurait perdu toute fluidité et serait comme figé.

Cet état amorphe bien que très courant dans la nature ne constitue pas un état thermodynamiquement stable. Ainsi un solide cristallin devient liquide lorsqu'on élève sa température (transition du $1^{\text {er }}$ ordre). Réciproquement le liquide cristallise lorsqu'on le refroidit. Cependant dans certaines conditions on peut 
former un verre et ses propriétés changent alors continûment. De plus les corps amorphes possèdent des propriétés physiques très particulières, le plus souvent incompatibles avec la structure polycristalline (la transparence du verre); ou encore ils présentent des associations de propriétés considérées comme antagonistes pour un polycristal (ferromagnétisme doux et de bonnes propriétés mécaniques).

D'un point de vue phénoménologique, l'usage était de définir cet état amorphe comme ne présentant pas de spectre de diffraction fin. Cette anomalie par rapport aux concepts introduits dans la physique des cristaux avait suscité l'intérêt des physiciens bien avant qu'on ait découvert leurs propriétés de commutations d'un état semiconducteur à un état métallique et on peut citer à ce sujet les travaux d'Anderson [1], de Kolomiets [2] et de Mott [3].

La principale difficulté dans l'étude et la compréhension de cet état provient de l'absence d'un référentiel. En effet la description des cristaux a pu être faite grâce aux distributions relativement fixes des atomes dans un espace à trois dimensions.

Par comparaison avec l'arrangement ordonné des atomes dans un cristal, les atomes ou les molécules d'un gaz sont caractérisés par un grand désordre. Les distributions des molécules autour de chacune d'entre elles sont uniformes puisque les mouvements sont relativement grands et se font essentiellement au hasard.

Pour les cas limites de gaz dilués comportant un désordre presque parfait ou bien pour des cristaux comportant un ordre presque parfait, les structures atomiques ou moléculaires sont souvent bien connues. Ainsi grâce à la grande distance moyenne entre atomes dans le cas d'un gaz et à l'ordre du réseau dans le cas d'un solide cristallin, on a pu faire des hypothèses simplificatrices qui ont permis d'établir les équations d'état.

La situation des amorphes est hélas bien moins satisfaisante car les hypothèses simplificatrices faites pour les gaz et les solides cristallins ne sont malheureusement plus valables. Néanmoins l'impossibilité de l'interpénétration des atomes et l'existence de forces de liaisons interatomiques impliquent que certains arrangements sont plus probables que d'autres. Il existe donc nécessairement un ordre à courte distance ou une structure caractéristique de chaque amorphe. Cette structure atomique dont la connaissance pourrait amener à une meilleure compréhension de cet état possède les caractéristiques suivantes : elle est statistiquement homogène et isotrope, toutes les directions jouant le même rôle, désordonnée à grande distance, mais cependant elle présente à courte distance un ordre local très marqué que nous nous proposons d'examiner en utilisant la fonction de corrélation de paire $g(r)$.

1. Fonction de corrélation de paire $\mathbf{g}(\mathbf{r})$. Définition. - Considérons d'une manière générale un ensemble d'atomes formant un système désordonné à une température $T$ et dont la densité atomique moyenne exprimée en nombre d'atomes par unité de volume est $\rho_{0}$. Si $\rho(r)$ est la densité atomique à la distance $r$ d'un atome quelconque choisi comme atome de référence, on peut définir la fonction de distribution de paire qui mesure les fluctuations de la densité par rapport à la densité moyenne par la simple relation :

$$
g(r)=\frac{\rho(r)}{\rho_{0}} .
$$

Si $r$ devient grand, le milieu apparaît comme isotrope $\rho(r) \rightarrow \rho_{0}$ et $g(r)$ devient égal à 1 . Une fonction de ce type est représentée sur la figure 1 . Cette fonction est

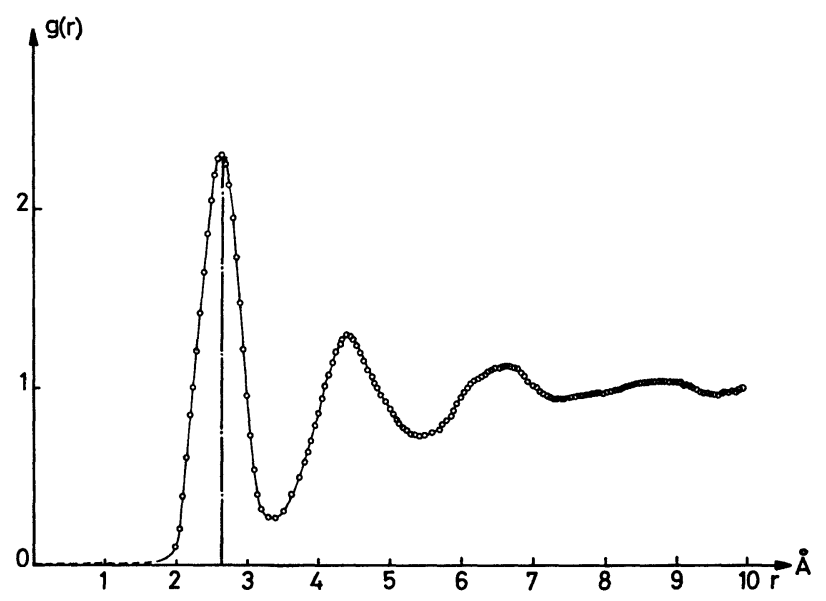

FIG. 1. - Fonction de corrélation de paire $g(r)$.

nulle pour les petites valeurs de $r$, c'est-à-dire $r>2 \AA$, car les atomes ne peuvent évidemment pas s'interpénétrer. Au voisinage du cœur dur $g(r)$ présente un maximum car les atomes ont tendance à se presser les uns contre les autres créant ainsi un encombrement qui empêche d'autres atomes de s'approcher d'où le minimum suivant et ainsi de suite.

La connaissance de la fonction $g(r)$ permet de calculer simplement les nombres de coordinance puisque ceux-ci sont reliés aux aires situées sous les pics de la fonction $4 \pi r^{2} \rho_{0} g(r)$. La figure 2 représente cette fonction et il est aisé de constater que l'aire sous le premier pic est très bien délimitée mais hélas il n'en est pas toujours ainsi. Il existe alors de nombreuses méthodes graphiques pour déterminer ces nombres suivant la plus ou moins grande séparation existant entre les différents pics de cette fonction. Cependant pour que les différentes valeurs obtenues soient comparables, il est bien évident qu'elles doivent être calculées de la même manière. Enfin ce nombre de coordinance n'est qu'une moyenne statistique temporelle et spatiale du nombre de premiers, seconds, ... voisins.

La détermination de $g(r)$ fournira aussi des renseignements précieux sur la position, éventuellement l'angle de liaison de ces plus proches voisins ainsi que sur les variations de ces grandeurs en fonction de paramètres tels que la température, la pression, ou bien 


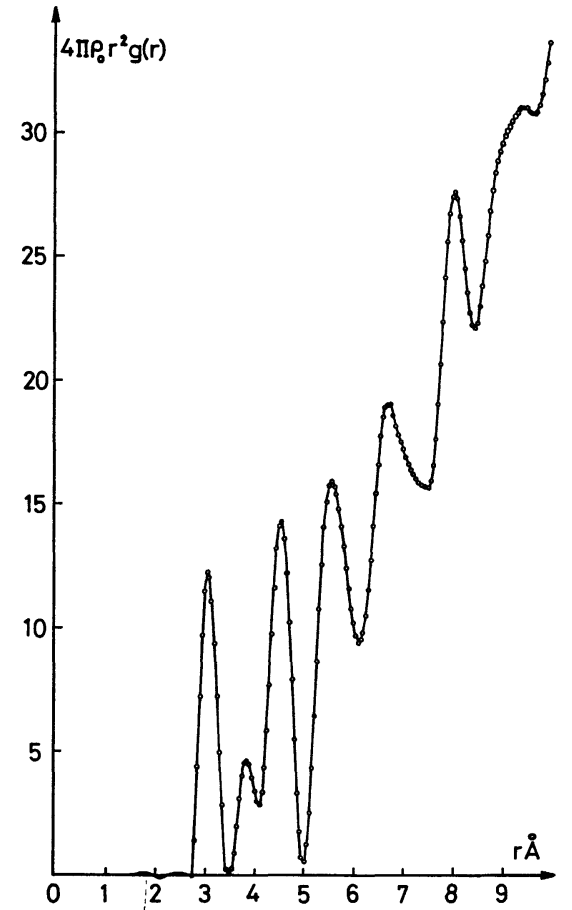

FIG. 2. - Fonction de distribution radiale $4 \pi \rho_{0} r^{2} g(r)$ utilisée pour le calcul des nombres de coordinance.

encore dans le cas des systèmes binaires : la concentration.

2. Diffusion de rayonnement et facteur de structure. - Pour déterminer cette fonction de corrélation de paire $g(r)$, on mesure le facteur de structure $S(k)$ dont elle est la transformée de Fourier. Cette mesure peut être déduite de l'intensité diffusée par un échantillon soumis à un rayonnement de longueur d'onde et d'énergie convenablement choisies.

Nous pouvons rappeler que d'une manière générale, chaque fois qu'un rayonnement monochromatique

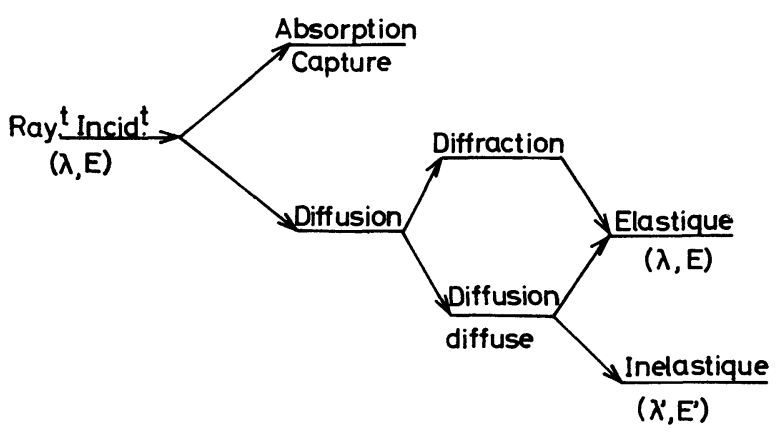

Fig. 3. - Processus de diffusion d'un rayonnement monochromatique.

(photons, électrons, neutrons, $\gamma$, etc...) : interagit avec un solide, on observe toujours (cf. Fig. 3) :

1) qu'une fraction plus ou moins importante du rayonnement disparaît, c'est le phénomène d'absorption ou de capture ;

2) qu'une autre fraction du rayonnement est plus ou moins déviée de sa trajectoire, c'est le phénomène de diffusion.

Ce rayonnement diffusé comprend deux parties pour lesquelles les caractères de la diffusion sont très différents ;

a) une partie est fortement concentrée dans des directions bien déterminées, liées d'ailleurs à la structure cristalline du solide. C'est le rayonnement diffracté. Les directions privilégiées où le rayonnement est ainsi concentré correspondent aux pics de Bragg ;

b) une autre partie du rayonnement est diffusée d'une manière sinon isotrope du moins suivant une fonction un peu plus douce de l'azimut que les pics de Bragg précédents. C'est la diffusion diffuse.

Si maintenant on effectue une analyse en longueur d'onde ou en énergie, ce qui revient au même, du rayonnement diffusé, on peut distinguer deux cas : ou bien le rayonnement diffusé a même énergie que le rayonnement incident et la diffusion est dite élastique; ou bien il y a eu changement d'énergie dans le processus de diffusion et la diffusion est dite alors inélastique.

Nous allons maintenant examiner quels sont les domaines d'utilisation des différents types de rayonnements et lesquels sont les mieux adaptés aux divers types d'études envisageables sur les solides non cristallins.

Nous utiliserons pour cela le tableau de la figure 4, ce tableau est une extension de celui présenté par

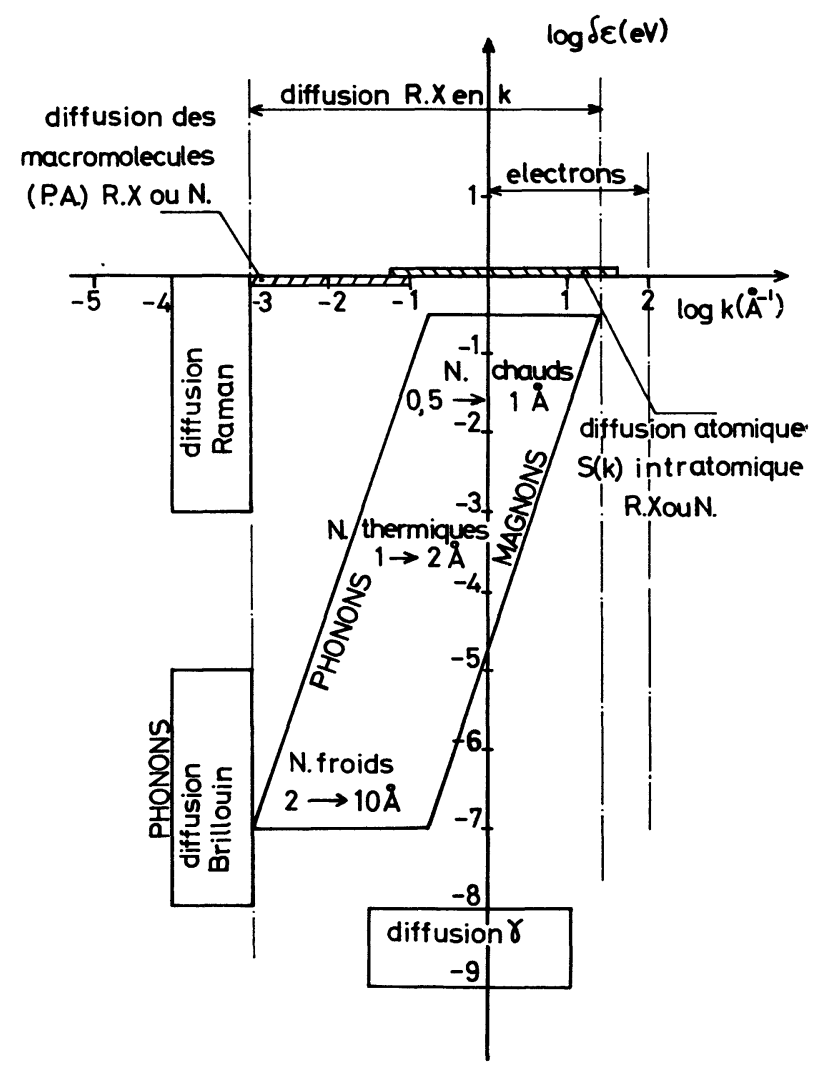

Fig. 4. - Localisation des domaines d'existence de divers rayonnements dans l'espace réciproque $k, \omega$. 
Egelstaff [4], il tient compte des possibilités expérimentales les plus récentes et le domaine de diffusion des rayons $\mathrm{X}$ et des électrons y a été mentionné. Le plan de l'espace réciproque $k \omega$ est ainsi représenté avec en abscisses les vecteurs de diffusion $k$ en $\AA^{-1}$ et en ordonnées les transferts d'énergie $\partial \varepsilon$ en $\mathrm{eV}$.

On peut constater que l'utilisation de la diffusion de la lumière, des rayons gamma, des rayons $\mathrm{X}$, des électrons et des neutrons permet d'obtenir des informations dans une grande partie du plan $k, \omega$ situé entre $10^{-4}$ et $100 \AA^{-1}$ en $k$, et entre $10^{-9}$ et $0,5 \mathrm{eV}$ en transfert d'énergie. Il nous faut remarquer que pour les rayons $\mathrm{X}$ et les électrons, les transferts d'énergie sont possibles dans une large gamme, mais ces transferts d'énergie ne sont pas mesurables (résolution beaucoup trop faible). A la base du tableau nous avons la diffusion $\gamma$ pour laquelle les transferts d'énergie n'occupent qu'un très faible domaine de $10^{-9}$ à $10^{-8} \mathrm{eV}$.

Pour l'étude de phénomènes mettant en jeu un transfert d'énergie, nous voyons que les rayonnements les plus utilisés seront par conséquent les photons dans le visible, soit par effet Brillouin, avec des transferts d'énergie de $10^{-8}$ à $10^{-5} \mathrm{eV}$, soit par effet Raman où les transferts d'énergie occupent la gamme de $10^{-3}$ à $1 \mathrm{eV}$. Nous n'aborderons pas ce sujet puisqu'il sera traité plus en détail dans d'autres exposés lors de ces journées d'étude sur la caractérisation des solides non cristallins.

Enfin au centre du tableau une zone relativement importante par son extension aussi bien en vecteur de diffusion qu'en transfert d'énergie, représente le domaine d'utilisation des neutrons. Nous voyons que la gamme de transfert d'énergie couverte s'étend de $10^{-7}$ à $0,5 \mathrm{eV}$ pour des vecteurs d'onde de $10^{-3}$ à $30 \AA^{-1}$.

Il faut noter que c'est seulement au cours des toutes dernières années que les valeurs extrêmes ont pu être atteintes grâce à l'utilisation de sources chaudes et de sources froides dans les réacteurs expérimentaux.

Les neutrons permettent donc l'étude de la dynamique ainsi que les excitations individuelles ou collectives à l'échelle atomique dans les systèmes condensés.

En ce qui concerne les études directes de la structure, nous devons remarquer tout d'abord que la diffusion de la lumière ne pourra nous apporter aucune information sur l'ordre local. En effet les longueurs d'onde utilisées conduisent à une gamme de vecteurs de diffusion de $10^{-3}$ à $10^{-4} \AA^{-1}$ alors que les premiers pics du facteur de structure atomique pour les corps amorphes se situent à quelques $\AA^{-1}$. On voit donc que les rayonnements utilisables pour l'étude de la diffusion atomique dans la gamme de $10^{-1}$ à $30 \AA^{-1}$, ou bien encore pour l'étude de la structure des macromolécules ou de groupement d'atomes, pour des vecteurs de diffusion de $10^{-3}$ à $10^{-1} \AA^{-1}$ sont essentiellement les rayons $\gamma$, les électrons, les rayons $\mathrm{X}$ et les neutrons.

Nous ne nous étendrons pas sur la diffusion par les rayons $\gamma$ car les flux extrêmement minimes en limitent l'emploi au domaine des monocristaux. Ils sont cepen- dant d'une grande utilité, dans le cadre de la diffusion à haute résolution, notamment pour déterminer la mosaïcité d'un monocristal. Mais cette haute résolution n'est pas très utile dans l'étude structurale des systèmes non cristallins.

Le cas des électrons est plus ambigu, en effet il est possible d'obtenir des flux relativement élevés mais on est très vite limité par les problèmes d'absorption. Si $\mu$ est le coefficient d'absorption linéaire, la profondeur de pénétration $x$, définie par $\mu x=1$ dans la relation d'absorption :

$$
I=I_{0} \mathrm{e}^{-\mu x}
$$

sera de l'ordre de $10^{2}$ à $10^{3} \AA$ suivant l'échantillon considéré.

Pour des électrons dont les énergies varient entre 50 et $100 \mathrm{keV}$ les longueurs d'onde sont respectivement de 5,2 et $3,7 \times 10^{-2} \AA$. Il est donc clair que pour rendre possible une étude structurale par la diffusion des électrons, il faut disposer de couches très minces, quelques centaines d'Angströms, et d'un système de détection possédant une résolution très élevée de l'ordre du 1/100 de degré. Cependant on peut réaliser des expériences de diffusion d'électrons lents ayant des longueurs d'onde de l'ordre de 1,4 $\AA$ (ce qui correspond à des énergies de $75 \mathrm{eV}$ ). Dans ces expériences, la longueur d'onde est bien cette fois du même ordre de grandeur que les distances interatomiques. Mais dans ce cas, la profondeur de pénétration n'excède guère une couche atomique, c'est-à-dire 2 à $3 \AA$, de telles expériences peuvent conduire à l'étude structurale des surfaces.

3. Comparaison rayons $X$. Neutrons. - Nous allons maintenant examiner plus en détail le cas des rayons $\mathrm{X}$ et des neutrons. La différence essentielle entre ces deux rayonnements réside dans la nature même de leur diffusion qui est électronique pour la première, tandis que la seconde est nucléaire. Ceci a pour conséquence importante que le facteur de diffusion des neutrons est isotrope, c'est-à-dire indépendant de l'angle de diffusion. C'est ainsi que pour des valeurs de $k$ élevées, l'intensité diffusée est constante, ce qui facilite considérablement la détermination du facteur de structure $S(k)$.

Les caractéristiques des faisceaux sont également différentes. En ce qui concerne les rayons X, le spectre émis par l'anticathode comprend un fond continu sur lequel se détachent les radiations $\mathrm{K} \alpha, \mathrm{K} \beta$, caractéristiques de cette anticathode, avec une intensité très élevée par rapport au fond continu. Au contraire, le spectre des neutrons d'un réacteur est un spectre de Maxwell-Boltzmann qui ne présente pas l'analogue des radiations caractéristiques précédentes (Fig. 5). Tout se passe en fait comme si l'on avait que du fond continu.

Pour obtenir une radiation monochromatique intense aux rayons $\mathrm{X}$, il suffit de choisir un monochro- 

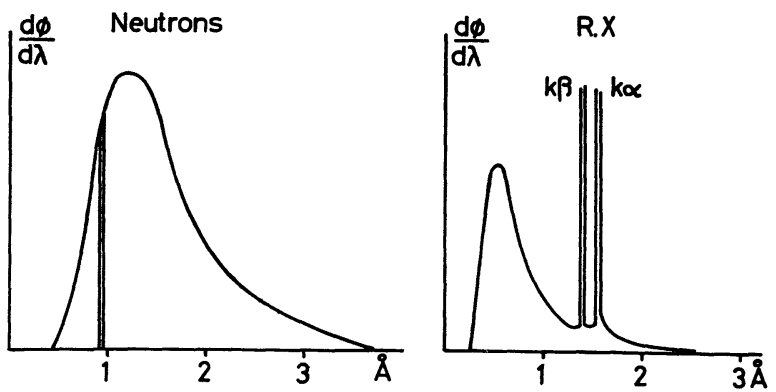

FIG. 5. - Distribution spectrale des intensités émises dans le cas des neutrons et des rayons $\mathbf{X}$.

mateur à cristal (généralement un quartz) qui réfléchisse une bande ne comprenant que l'étroite et intense raie d'émission $K \alpha$.

Par contre pour obtenir des neutrons monochromatiques, on sélectionnera par un monochromateur à cristal une bande du spectre au voisinage de la longueur d'onde $\lambda_{0}$ désirée. Mais cette bande devra être suffisamment large si l'on veut avoir un tant soit peu d'intensité à cause de l'absence de raie d'émission, d'où l'emploi de monocristaux à large mosaïque.

Tout ceci naturellement au détriment de la définition en angle et en longueur d'onde et même ainsi les flux de neutrons monochromatiques obtenus sont bien plus faibles que pour les rayons $X$.

Le tableau I résume les caractéristiques principales de ces deux rayonnements après monochromation

TABleaU I

\begin{tabular}{|c|c|c|c|}
\hline & $\Delta \lambda / \lambda$ & Divergence & Flux \\
\hline R.X & $10^{-4}$ & q.qs minutes d'arc & $10^{10}$ photons $/ \mathrm{cm}^{2} / \mathrm{s}$ \\
\hline Neutrons & $10^{-2}$ & le demi-degrè & 6 neutrons $/ \mathrm{cm}^{2} / \mathrm{s}$ \\
\hline
\end{tabular}

mais elle ne donne que des ordres de grandeur. Cependant, on peut constater que la résolution, la divergence angulaire ou le flux, toutes ces valeurs sont en faveur des rayons X. Dès lors on peut s'interroger sur l'intérêt que présente l'utilisation des neutrons par rapport aux rayons $X$.

La réponse est simple : c'est que les neutrons à quelques rares exceptions sont très peu absorbés par la matière. Ceci se comprend d'ailleurs aisément puisque le neutron est une particule électriquement neutre ce qui lui permet de passer au voisinage des atomes sans interagir avec leur charge électrique (électrons ou protons). Pour donner un ordre de grandeur, les coefficients d'absorption des solides sont d'environ $100 \mathrm{~cm}^{-1}$ pour les rayons $\mathrm{X}$ et $0,1 \mathrm{~cm}^{-1}$ pour les neutrons, c'est-à-dire 1000 fois moins, ce qui permet de regagner largement ce catastrophique facteur $10^{4}$ existant sur le flux.

On fait donc diffracter ou diffuser les neutrons par des échantillons d'un volume de plusieurs $\mathrm{cm}^{3}$ alors qu'avec les rayons $X$ on ne peut plus augmenter l'intensité dès que le volume dépasse le $\mathrm{mm}^{3}$. Ceci présente l'avantage considérable de s'affranchir au maximum des effets de surface tant sur le plan de la définition géométrique que sur celui des interactions chimiques.

Autre avantage de la nature nucléaire du procédé de diffusion des neutrons, c'est que les longueurs de diffusion varient dans de très larges limites d'un noyau à l'autre et en particulier d'un isotope à l'autre d'un même élément; on voit donc tout l'intérêt des méthodes spectrométriques en diffusion élastique ou inélastique des neutrons. C'est pourquoi le nombre d'études réalisées à l'aide des sources à neutrons est en progression constante.

De plus il est possible de déplacer le maximum du spectre de Maxwell-Boltzmann puisque le maximum de distribution des vitesses est donné par $V_{0}=\sqrt{2 k T} / m_{\mathrm{n}}$; en changeant la température à laquelle les neutrons sont thermalisés. L'emploi de plus en plus répandu de sources chaudes et froides sur le trajet des neutrons permet d'enrichir le spectre blanc en neutrons chauds (courte longueur d'onde) ou en neutrons froids (grande longueur d'onde).

Les flux de neutrons au niveau de l'échantillon sont de ce fait très variables d'un appareillage à un autre pour un même réacteur expérimental $[5,6]$. Enfin la possibilité d'avoir des faisceaux monochromatiques de neutrons polarisés, offre une technique d'étude d'une grande sensibilité, en particulier dans le cas des composés amorphes métalliques pouvant présenter des propriétés magnétiques.

4. Détermination de la fonction $\mathbf{g}(\mathbf{r})$. - Considérons un échantillon amorphe irradié par un faisceau monocinétique de rayons $X$ ou de neutrons, ayant une longueur d'onde comparable aux distances interatomiques. On conçoit bien que $g(r)$ qui décrit l'arrangement des atomes dans le solide va déterminer la distribution angulaire de l'intensité diffusée.

Si $\lambda$ est la longueur d'onde du rayonnement incident et $\theta$ l'angle de Bragg, le module du vecteur de diffusion sera défini par $k=4 \pi \sin \theta / \lambda$.

Ainsi on pourra mesurer dans le cas des neutrons thermiques par exemple, une fonction $S(k)$ de l'intensité diffusée par la relation :

$$
S(k)=\frac{I_{\mathrm{coh}}(k)}{I_{\mathrm{coh}}(\infty)},
$$

où $I_{\text {coh }}(k)$ représente la partie cohérente de l'intensité diffusée et $I_{\text {coh }}(\infty)$ est la valeur que prend $I_{\text {coh }}(k)$ lorsque $k$ est suffisamment grand pour que les effets d'interférence ne puissent plus être observés.

$I_{\text {coh }}(\infty)$ devient constant et on normalisera en donnant à cette constante une valeur telle que

$$
\lim _{k \rightarrow \infty} S(k)=1 \text {. }
$$

D'autre part, la thermodynamique statistique relie 
les fluctuations du nombre d'atomes contenus dans un élément de volume de grandeur donnée, au coefficient de compressibilité isotherme :

$$
\beta=-\frac{1}{V}\left(\frac{\partial V}{\partial p}\right)_{T}
$$

et $S(0)$ qui mesure ces fluctuations peut donc s'écrire

$$
S(0)=k_{\mathrm{B}} T \rho_{0} \beta
$$

où $k_{\mathrm{B}}$ est la constante de Boltzmann et $\beta$ le coefficient de compressibilité isotherme du système.

Il existe alors une relation exacte qui relie $S(k)$ et $g(r)$ par transformation de Fourier et on peut écrire :

$g(r)=\frac{\rho_{r}}{\rho_{0}}=1+\frac{1}{2 \pi^{2} \rho_{0} r} \int_{0}^{\infty} k[S(k)-1] \sin k r \mathrm{~d} k$.

Il est clair que la mesure expérimentale de $S(k)$ permet de calculer $g(r)$. Cependant expérimentalement les choses sont un peu moins simples. Ainsi pour des neutrons d'environ $1 \AA$ de longueur d'onde $(60 \mathrm{meV})$ on ne peut déterminer $S(k)$ que pour des valeurs comprises entre $k_{\min } \simeq 0,1 \cdot \AA^{-1}$ et $k_{\max } \simeq 12 \AA^{-1}$.

Dans la plupart des expériences sur les corps amorphes des oscillations peuvent encore être observées au-delà de 10 ou $12 \AA^{-1}$ dans l'espace réciproque. La figure 6 montre dans le cas de l'Arsenic amorphe l'importance des oscillations à $10 \AA^{-1}$.

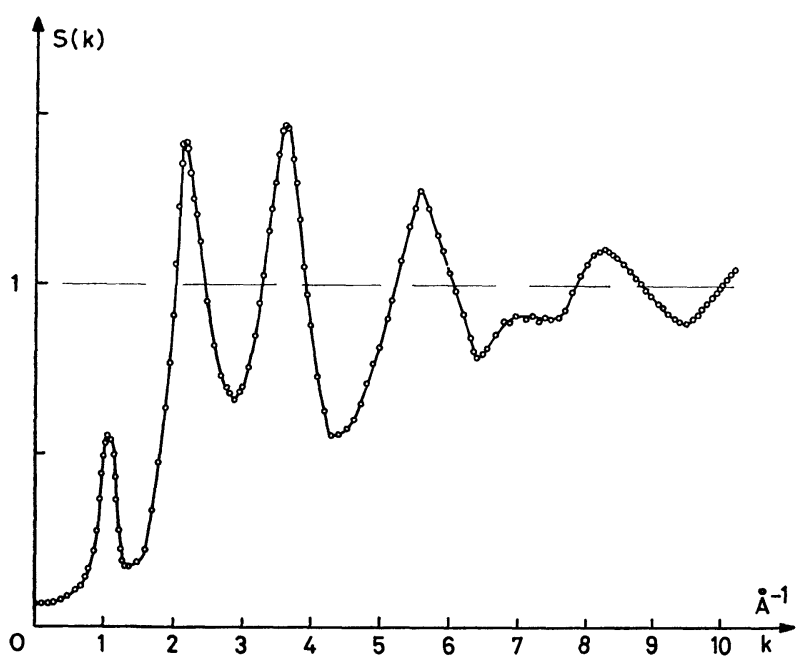

Fig. 6. - Facteur de structure de l'Arsenic amorphe.

Il sera donc souhaitable d'utiliser deux longueurs d'onde différentes, par exemple $1 \AA$ pour avoir une bonne détermination du facteur de structure aux petites valeurs de $k$, puis une longueur d'onde de $0,5 \AA$ qui permet d'atteindre des valeurs du vecteur de diffusion de $25 \AA^{-1}$.

Dans le cas d'études de systèmes où les corrélations à très courte distance sont importantes, il peut s'avérer utile de disposer de sources permettant d'atteindre des valeurs de $k$ encore plus élevées. On peut citer pour les rayons $\mathrm{X}$ les anneaux de collision d'Orsay $L U R E$ qui permettent d'atteindre quelques centaines d' $\AA^{-1}$ [7].

Pour les neutrons l'utilisation d'un accélérateur linéaire de type Linac en Grande-Bretagne permet de dépasser $35 \AA^{-1}$. Une seconde version fournira prochainement des neutrons dont le vecteur de diffusion atteindra environ $70 \AA^{-1}$.

5. Conclusion. - Nous venons de voir qu'une bonne connaissance du facteur de structure $S(k)$ permettait de déterminer la fonction de corrélation de paire $g(r)$ d'en déduire les nombres de coordinance ainsi qu'un certain nombre d'autres informations sur l'ordre local.

Une démarche semblable dans le cas des systèmes binaires permettra de déterminer les fonctions $g(r)$ partielles caractéristiques de chaque sorte de paire en fonction des facteurs de structure partiels. La mesure de ces facteurs de structure partiels peut être réalisée en utilisant différentes méthodes.

La première fondée sur la variation des concentrations permet d'atteindre les trois fonctions de distribution radiale, mais elle implique l'hypothèse rarement justifiée de l'invariance des facteurs de structure partiels en fonction de la concentration.

La seconde consiste à utiliser des rayonnements différents, rayons $\mathrm{X}$ et neutrons par exemple (méthode à deux diagrammes). Enfin l'effet isotopique dans le cas des neutrons, semble être actuellement la meilleure méthode d'étude des systèmes binaires.

Toutefois la connaissance de ces fonctions de corrélation ne permet pas de définir complètement l'ordre local dans le système puisque par définition elles sont isotropes et ne prennent en compte que les interactions de paire. Il est clair que l'on ne pourra pas en déduire toutes les propriétés physiques du système. Une démarche possible consiste à calculer à partir d'un modèle les fonctions de corrélation de paire et à en déduire les valeurs des paramètres physiques afin de les comparer aux données expérimentales. C'est pourquoi si la fonction de distribution radiale $g(r)$ est d'une grande importance pour la détermination de l'ordre à courte distance, elle ne pourra conduire à une connaissance approfondie de cet ordre local, qu'utilisée conjointement avec les autres propriétés physiques du système.

\section{Bibliographie}

[1] Anderson, P. W., Phys. Rev. 109 (1958) 1492

[2] Kolomiets, B. T., Phys. Stat. Sol. 7 (1964) 359, 713.

[3] MotT, N. F., Phil. Mag. 17 (1968) 1259.

[4] Egelstaff, P. A., An Introduction to the Liquid State (Academic Press) 1967.

[5] Briggs, G. A. and Stirling, W. G., Neutron Diffraction
Facilities at Western European Medium Flux Reactors, ILL Grenoble (1976).

[6] Neutron Beam Facilities, ILL High Flux Reactor (1973).

[7] WuIlleumier, F., Caractéristiques du Rayonnement Emis par les Anneaux de Collision d'Orsay (ACO et DCI) Lure-Orsay (1973). 